\title{
Cuantificación del Contenido de Carbono en una Plantación de Pino Insigne (Pinus radiata) y en Estrato de Páramo de Ozogoche Bajo, Parque Nacional Sangay, Ecuador
}

\author{
Franklin E. Cargua, Marco V. Rodríguez, Celso G. Recalde y Luis M. Vinueza \\ Universidad Nacional de Chimborazo (UNACH); Av. Antonio José de Sucre Km 11/2 vía a Guano; \\ Riobamba-Ecuador (e-mail: franklincargua@hotmail.com; marviny_rodriguez@yahoo.es; \\ crecalde672000@yahoo.com; marcelvinueza@hotmail.com)
}

Recibido Nov. 8, 2013; Aceptado Ene. 10, 2014; Versión final recibida Feb. 4, 2014

\begin{abstract}
Resumen
Se ha determinado el contenido de carbono orgánico en biomasa aérea, sotobosque, detritus no vivo, raicillas y suelo, en una plantación de Pinus radiata y en páramo, ubicado en la zona sur occidental del Parque Nacional Sangay, en Ecuador; se establecieron seis parcelas permanentes de muestreo. El contenido de carbono promedio para biomasa aérea en la plantación fue de $14.69 \mathrm{MgC} / \mathrm{ha}$, sotobosque $0,68 \mathrm{MgC} / \mathrm{ha}$, detritus no vivo $2.97 \mathrm{MgC} / \mathrm{ha}$, raicillas $0.15 \mathrm{MgC} / \mathrm{ha}$ y suelo $268,83 \mathrm{MgC} / \mathrm{ha}$. En páramo el valor promedio para sotobosque fue de $6.55 \mathrm{MgC} / \mathrm{ha}$, detritus no vivo $56.09 \mathrm{MgC} / \mathrm{ha}$, raicillas $0,17 \mathrm{MgC} / \mathrm{ha}$ y suelo $215.0 \mathrm{MgC} / \mathrm{ha}$, el carbono promedio total en la plantación es de $287.31 \mathrm{MgC} / \mathrm{ha}$ y en páramo $277.81 \mathrm{MgC} / \mathrm{ha}$, observando un incremento del $30 \%$, en la plantación de Pinus radiata, pero con una menor tasa de regeneración de especies herbáceas y arbustivas con relación al páramo. Los resultados del contenido de carbono presente en el área de estudio son similares a los encontrados en los páramos de provincias cercanas reportados en la literatura.
\end{abstract}

\section{Quantification of Carbon in a Forest Plantation of Pine Insigne (Pinus radiata) and Moor Stratum of Low Ozogoche, Sangay National Park, Ecuador}

\begin{abstract}
The organic carbon content in aerial and underground biomass, low stratum, litter, rootlets and soil in both Pinus radiata plantation and moor in the south western area of Sangay National Park, in Ecuador, has been determined. Six permanent sample plots were established. In the forest plantation, aerial biomass carbon content average value was $14.69 \mathrm{MgC} / \mathrm{ha}, 0,68 \mathrm{MgC} / \mathrm{ha}$ for low stratum, $2.97 \mathrm{MgC} / \mathrm{ha}$ for litter, $0.15 \mathrm{MgC} / \mathrm{ha}$ for rootlets and $268.83 \mathrm{MgC} / \mathrm{ha}$ for soil. In the moor, low stratum carbon content average value was 6.55 $\mathrm{MgC} / \mathrm{ha}, 56.09 \mathrm{MgC} / \mathrm{ha}$ for litter, $0.17 \mathrm{MgC} / \mathrm{ha}$ for rootlets and $215.0 \mathrm{MgC} / \mathrm{ha}$ for soil. Total average carbon was $287.31 \mathrm{MgC} / \mathrm{ha}$ in plantation and $277.81 \mathrm{MgC} / \mathrm{ha}$ in moor, an increase of $30 \%$ in Pinus radiata plantation relative to moor is observed but with a lower regeneration rate of herbaceous and shrub species. Results indicate that values of the carbon content in the area considered in the study are similar to values reported in the literature for other moors of neighboring provinces.
\end{abstract}

Keywords: carbon, biomass, soil, forest plantation, moor 


\section{INTRODUCCIÓN}

El páramo es un ecosistema neo tropical ubicado en Centro y Suramérica, en la franja entre el bosque montano y el límite superior de la nieve perpetua, es decir, aproximadamente entre 3000 y 5000 msnm, (Sklenáŕ et al., 2005). Se caracteriza por ser un ambiente frío y húmedo, con temperaturas diarias que fluctúan entre los $-3^{\circ} \mathrm{C} \mathrm{y}+20^{\circ} \mathrm{C}$ (Mena y Balslev, 1986) y una precipitación anual que varía desde 500 hasta sobre 3000 mm (Luteyn, 1999). En términos biológicos, los páramos constituyen una parte importante de la diversidad ecológica del Ecuador, (Medina et al., 1997; Medina, 2000; Medina y Mena, 2001), pero con una variedad ambiental y biológica mayor a la de países con extensiones muy superiores (Mittermeier et al., 1997). Los suelos de los páramos del Ecuador se han desarrollado esencialmente sobre los depósitos piroclásticos resultantes de las erupciones de los numerosos edificios volcánicos (Quantin y Zebrowski, 1997). Se trata en general de andosoles, pero su morfología y propiedades varían considerablemente según los principales factores de la pedogénesis, como la edad, naturaleza, composición química de los materiales y las condiciones climáticas.

La acumulación de carbono orgánico total en el suelo (COTS) es un proceso importante para mitigar efectos del cambio climático (Houghton y Woodwell, 1989), ya que el suelo, además de ser un sumidero, es un reservorio de carbono estabilizado (Sánchez et al., 2011). Otro de los ecosistemas que ayudan al secuestro de carbono es la biomasa de los bosques, que con un manejo adecuado, pueden secuestrar más carbono (Sampaio, 2009), que otros ecosistemas terrestres, contribuyendo a la mitigación del cambio climático, producido por la degradación y deforestación. Se considera que los bosques podrían ser considerados como sumideros de carbono, y los dueños podrían recibir un pago por la venta de bonos de carbono en base al acuerdo de los diferentes protocolos internacionales e iniciativas nacionales (Masera, 1995; Návar, 2009). Por otra parte, después del Protocolo de Kyoto (1997), la reducción de gases de efecto invernadero ha llegado a ser una prioridad para la mayoría de los países (Hernández et al., 2006), las actividades forestales como la forestación y reforestación (Vesterdal et al., 2007), pueden ser implementadas para mitigar el calentamiento global (Hernández et al., 2006).

El dióxido de carbono $\left(\mathrm{CO}_{2}\right)$ es uno de los principales gases que contribuyen al incremento de la temperatura atmosférica (Torres et al., 2011), las concentraciones producidas por las actividades antrópicas como el cambio en los usos del suelo (Carvajal et al., 2009 y Fabre et al., 2010), afecta de forma negativa los procesos ecológicos, económicos y sociales además de sus efectos subsecuentes en los ecosistemas (Mascha et al., 2013), como el cambio de hábitats y perdida en la biodiversidad. Una forma de mitigar los efectos que genera el dióxido de carbono $\left(\mathrm{CO}_{2}\right)$ es capturarlo y mantenerlo el mayor tiempo posible en la biomasa de las plantas (Pauli et al., 2003) y en el suelo (Torres et al., 2011). Por esta razón se han implementado políticas gubernamentales y acuerdos internacionales que proporcionan mecanismo de flexibilidad; uno de ellos el proyecto llamado mecanismo de desarrollo limpio (Concha et al., 2007), como forma de compensar a los países que protegen sus bosques y aquellos que reducen las emisiones de gases de efecto invernadero (GEI), (Torres et al., 2011).

La estimación precisa de la dinámica de los flujos netos de carbono entre los bosques y la atmósfera (es decir, el balance emisión-captura) es uno de los problemas abiertos más importantes en la discusión sobre cambio climático (IPCC, 2005). El objetivo del presente trabajo fue estudiar el almacenaje de carbono en los cinco depósitos propuesto por el IPCC (2006), en una plantación de pino (Pinus radiata), establecida en un ecosistema de páramo (Hofstede, 2000). Los resultados del contenido de carbono presente en el área de estudio son similares a los encontrados en los páramos de provincias cercanas como: Carchi, Cañar, Azuay, Pichincha y Loja, con valores de 463, 466, 364, 479, 356 y $239 \mathrm{MgC} / \mathrm{ha}$, respectivamente (Hofstede et al., 2003).

\section{MATERIALES Y MÉTODOS}

\section{Área de Estudio}

La presente investigación se llevó a cabo en la comunidad de Ozogoche Bajo, que se encuentra ubicada en la zona sur occidental del parque Nacional Sangay en la parroquia Achupallas, cantón Alausí, provincia de Chimborazo, Ecuador en una plantación de pino (Pinus radiata) que abarca una superficie de 100 ha; las muestras se tomaron en rangos de altitud desde los 3792 - $4096 \mathrm{msnm}$. Los datos obtenidos de los anuarios de la estación meteorológica (M396 INHAMI 2010); presentan una temperatura media de $8{ }^{\circ} \mathrm{C}$, con rangos de $(4-12)^{\circ} \mathrm{C}$; una precipitación de 1077 con rangos de $(1000-1200) \mathrm{mm}$. 
En la zona de estudio podemos encontrar especies endémicas Cortaderia nítida, Calamagrostis intermedia, Hypochaeris sessiliflora, Wernerianubigena, (Luteyn, 1999; Jorgensen y León-Yánez, 1999; MenaVásconez, 2001; León-Yánez, 2000), Para la investigación se ha considerado la comparación del contenido de carbono en una plantación de pino (Pinus radiata), en estado fustal de 18 años de edad, establecido a una altura de $3840 \mathrm{msnm}$; y el contenido de carbono presente en un ecosistema de páramo ubicado a una altura de $4000 \mathrm{msnm}$.

\section{Muestreo}

Se aplicó un diseño de muestreo de doble estratificación, el cual consiste en dos fases. Para la primera fase se definió una rejilla de puntos de (1x1) km con proyección geográfica UTM WGS'84 Zona 17S; path-row 10-62 (glovis.usgs.gov), luego se seleccionó una muestra más pequeña en dos estratos correspondientes a seis parcelas de 60 x $60 \mathrm{~m}$ (Fig. 1); (Cueva et al., 2012).

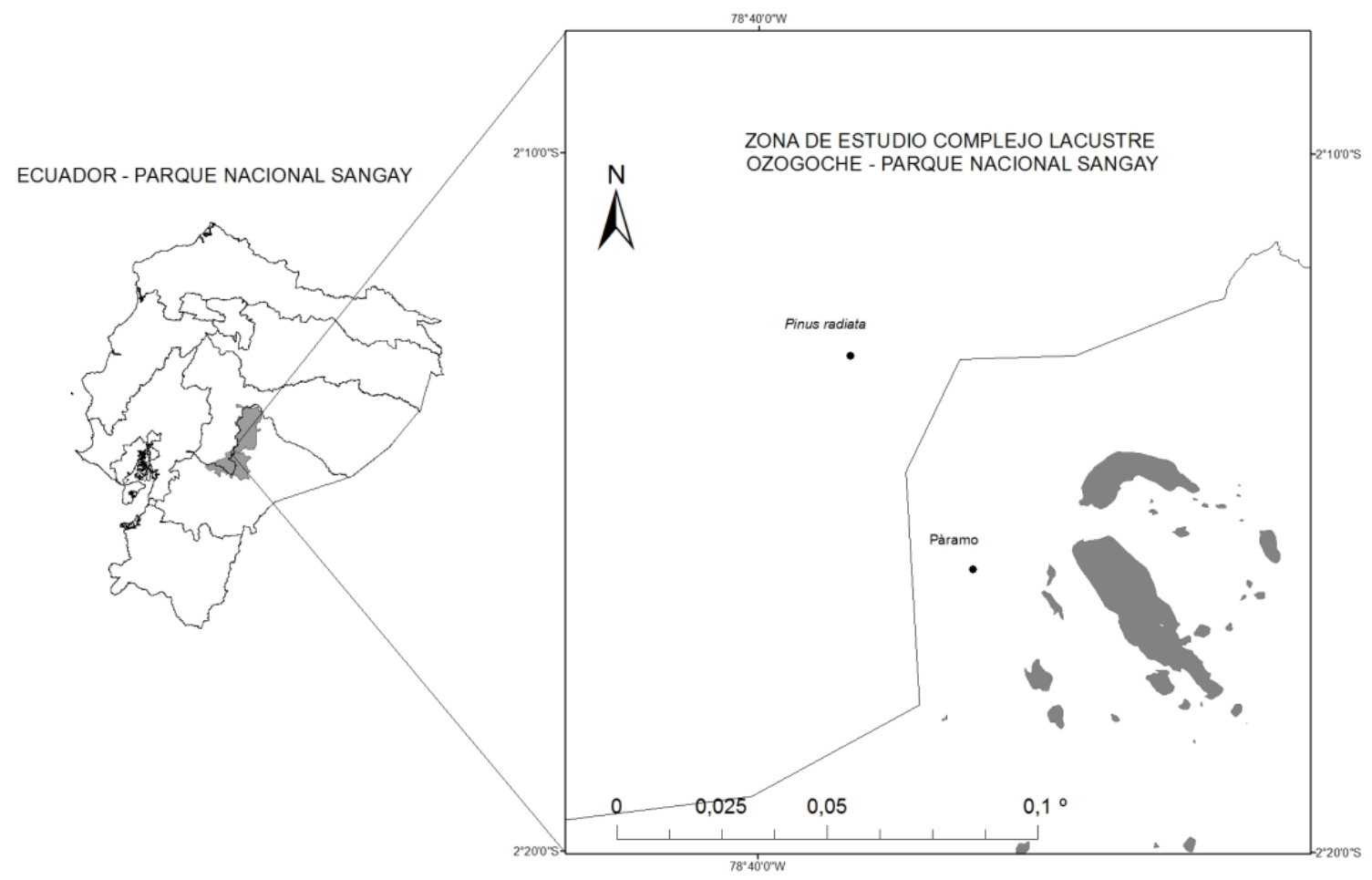

Fig. 1: Ubicación de las parcelas permanentes de muestreo

Estratificación de unidades de muestreo

Para las mediciones de campo se utilizó un conglomerado de parcelas en forma de L. Las parcelas fueron de forma cuadrada de $60 \times 60 \mathrm{~m}$ (Fig. 2). Esta forma de parcelas es usual para las mediciones de biomasa y la mayoría de tipos de vegetación (Ravindranath y Oswald, 2008).

Ecuación 1. Para determinar (i) intensidad de muestra.

$i=\frac{\text { superficie de la muestra }}{\text { superficie total }} \times 100$

En cada muestra seleccionada se midieron y observaron todas las variables de interés como: altura total, diámetro a la altura del pecho, biomasa aérea y subterránea, sotobosque, detritus no vivo y suelo. En la parcela de $60 \times 60 \mathrm{~m}$, se realizó un inventario forestal, se estableció una sub-parcelas de $20 \times 20 \mathrm{~m}$, para recolectar muestras de suelo a tres profundidades: entre (0-10), (10-20), (20-30) cm; se implementó una segunda sub-parcela de $50 \times 50 \mathrm{~cm}$, donde se procede a colectar todo el material muerto y registrar su peso húmedo. La tercera sub-parcela es de $2 \times 2 \mathrm{~m}$ y permite la medición de sotobosque que para el caso de páramo corresponde todo la parte herbácea. 


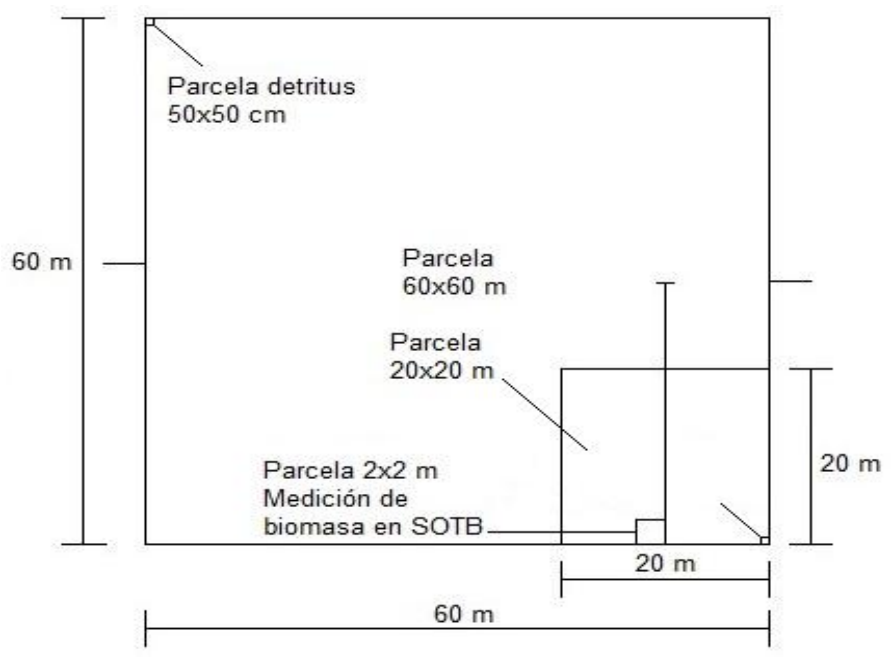

Fig. 2: Diseño, distribución y tamaños de las parcelas anidadas para las mediciones

Para determinar el contenido de biomasa aérea, se utilizó factores de expansión, para fuste + copa 2,8, raíz 0,27 y densidad de la madera $0,54 \mathrm{~g} / \mathrm{cm}^{3}$, se refiere a un coeficiente que utilizan los volúmenes o existencias reales provenientes de los inventarios forestales para estimar biomasa y carbono (Chávez et al., 2013, Silva y Navar, 2009). A partir de ellas se ha generado mucha información que constituye una base útil a la hora de realizar estimaciones de la capacidad de fijación de carbono en un bosque.

\section{RESULTADOS Y DISCUSIONES}

\section{Establecimiento de las parcelas permanentes de muestreo}

En la tabla 1 muestra el número de conglomerados y la cantidad de parcelas distribuidas en cada uno de los sectores del área de estudio, las mismas que están debidamente georeferenciadas y ubicadas en sitios donde es posible realizar el levantamiento de información (monitoreo).

Tabla1. Localización de las Parcelas Permanentes de Muestreo (PPM)

\begin{tabular}{cccccc}
\hline Conglomerado & Parcela & Descripción & Este & Norte & Altitud (msnm) \\
\hline \multirow{2}{*}{001} & P1 & Páramo conservado & 765171 & 9749347 & 4096 \\
& P2 & Páramo conservado & 764921 & 9749347 & 3984 \\
& P3 & Páramo conservado & 764921 & 9749597 & 4020 \\
\hline \multirow{2}{*}{002} & P1 & Pino (Pinus radiata) & 761931 & 9754976 & 3796 \\
& P2 & Pino (Pinus radiata) & 761681 & 9754976 & 3880 \\
& P3 & Pino (Pinus radiata) & 761681 & 9755227 & 3876 \\
\hline
\end{tabular}

\section{Inventario forestal}

Los bosques en crecimiento son capaces de una absorción neta de $\mathrm{CO}_{2}$ y la velocidad de absorción de $\mathrm{CO}_{2}$ es directamente proporcional a dicho crecimiento (Kyrklund, 1990). En la plantación de pino la densidad de siembra es de $3 \times 3 \mathrm{~m}$, obteniendo un total de 1111 plantas/ha, cabe mencionar que en el lugar no se ha realizado ningún tipo de manejo silvicultural. El inventario forestal en la plantación de pino, está distribuido por tres clases diamétricas, con un diámetro promedio de $0,22 \mathrm{~m}$, una altura promedio de $8,04 \mathrm{~m}$, una área basal promedio de $0,036 \mathrm{~m}^{2}$ y un volumen promedio de $0,18 \mathrm{~m}^{3}$, por árbol.

Tabla 2. Inventario de la plantación de Pinus radiata para determinar el contenido de carbono. * Media \pm Desviación estándar

\begin{tabular}{cccccc}
\hline $\mathrm{N}^{0}$ & Clase diamétrica $(\mathrm{cm})$ & DAP $\dot{\mathrm{X}}(\mathrm{m})$ & Altura total $\dot{\mathrm{X}}(\mathrm{m})$ & Área basal $\dot{\mathrm{X}}\left(\mathrm{m}^{2}\right)$ & Volumen total con corteza $\dot{\mathrm{X}}\left(\mathrm{m}^{3}\right)$ \\
\hline 1 & $10-15$ & 0,13 & 6,64 & 0,01 & 0,06 \\
2 & $>15-20$ & 0,18 & $7,88^{\star} \pm 0,34$ & $0,03^{*} \pm 0,01$ & $0,15^{\star} \pm 0,01$ \\
3 & $>20-25$ & $0,22^{\star} \pm 0,01$ & $9,04^{\star} \pm 0,71$ & 0,04 & $0,24^{*} \pm 0,02$ \\
4 & $>25-30$ & 0,28 & 9,56 & 0,06 & 0,41 \\
\hline
\end{tabular}




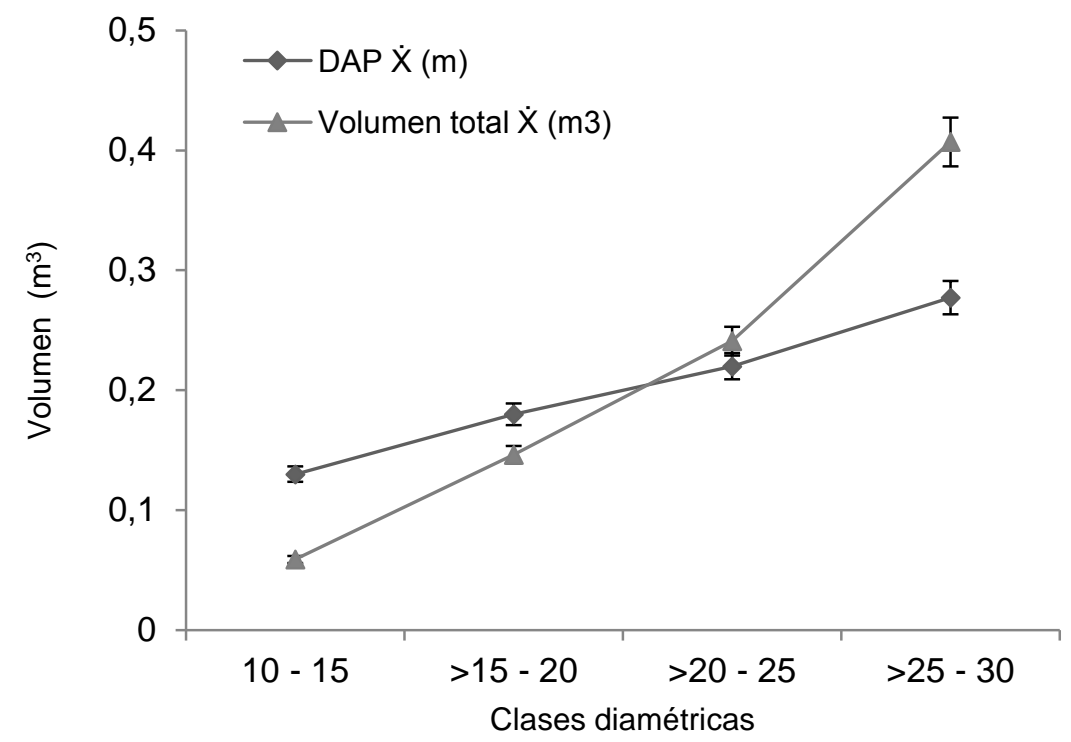

Fig. 3: Relación entre DAP y volumen total

\section{Contenido de carbono de la biomasa aérea y subterránea en la plantación de Pinus radiata}

Se ha realizado estudios especializados que han determinado factores estándar, los cuales nos han servido para determinar el contenido de carbono fijado en la plantación (Cueva et al., 2012). La variación del contenido de carbono orgánico total está representada por clases diamétricas en la plantación de pino, con un valor promedio de 0,20 MgC/ha con niveles de confiabilidad de 0,4-0,1 y una desviación estándar de $\pm 0,14$, los valores de biomasa aérea más alta se observaron en la clase diamétrica $(>20-25) \mathrm{cm}$, con un valor de 0,32 y una desviación de $\pm 0,04$. Los valores más altos de biomasa subterránea se observaron en la clase diamétrica $(>20-25) \mathrm{cm}$, con un valor medio de 0,09 con una desviación estándar de $\pm 0,01$; otros estudios sobre la biomasa de raíces de coníferas muestran valores entre 0,1 y 0,25 del árbol (Guerra et al., 2005)

Tabla 3. Contenido de biomasa aérea en la plantación de Pinus radiata

\begin{tabular}{ccc}
\hline Conglomerado & Parcela & Carbono $(\mathrm{Mg} / \mathrm{ha})$ \\
\hline \multirow{3}{*}{002} & P1 & 16,33 \\
& P2 & 11,03 \\
& P3 & 16,72 \\
\hline
\end{tabular}

Tabla 4. Relación entre biomasa aérea y subterránea de la plantación de Pinus radiata. * Media \pm Coeficiente variación

\begin{tabular}{cccccc}
\hline $\mathrm{N}^{0}$ & $\begin{array}{c}\text { Clase diamétrica } \\
(\mathrm{cm})\end{array}$ & $\begin{array}{c}\text { Biomasa } \\
\text { aérea }(\mathrm{Mg} / \mathrm{ha})\end{array}$ & $\begin{array}{c}\text { Biomasa } \\
\text { subterránea } \\
(\mathrm{Mg} / \mathrm{ha})\end{array}$ & $\begin{array}{c}\text { Biomasa } \\
\text { Total }(\mathrm{Mg} / \mathrm{ha})\end{array}$ & $\begin{array}{c}\text { Carbono Total } \\
(\mathrm{Mg} / \mathrm{ha})\end{array}$ \\
\hline 1 & $10-15$ & 0,09 & 0,02 & 0,11 & 0,06 \\
2 & $>15-20$ & 0,22 & 0,06 & 0,28 & 0,14 \\
3 & $>20-25$ & $0,36^{\star} \pm 0,04$ & $0,10^{\star} \pm 0,01$ & $0,46^{\star} \pm 0,05$ & $0,23^{\star} \pm 0,02$ \\
4 & $>25-30$ & 0,62 & 0,17 & 0,78 & 0,39 \\
\hline
\end{tabular}

Lazore y Gilabert, 2007, menciona dentro de sus estudios la relación entre raíz/fuste fue de 20,3 y 79,7\% respectivamente, el desarrollo de la plantación que varía con el tiempo pero a determinada edad el incremento se detiene. 


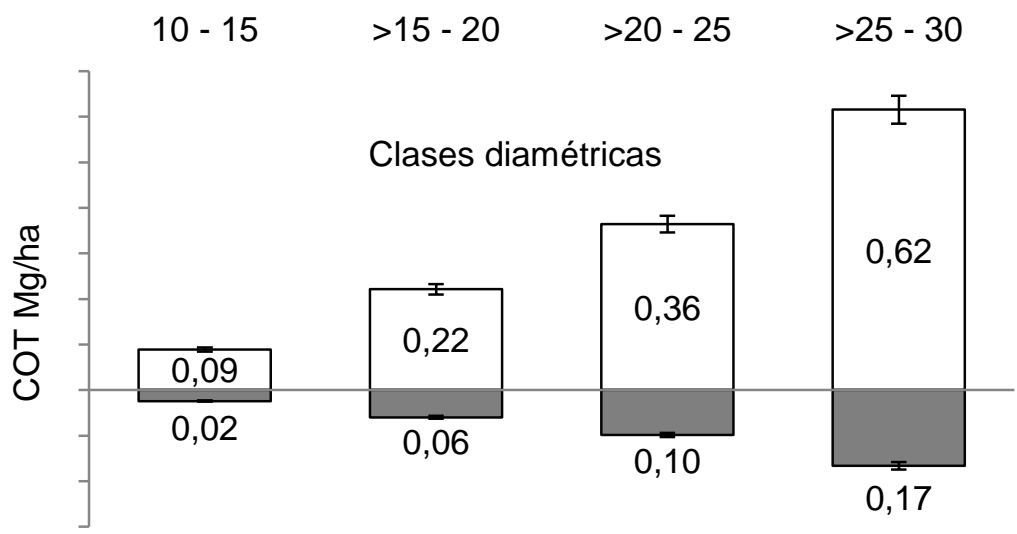

Fig. 4: Relación entre biomasa aérea $(\square)$ y biomasa subterránea

\section{Carbono presente a diferentes profundidades en las parcelas permanentes de muestreo (PPM)}

El contenido de carbono en el suelo fue mayor a una profundidad de $(20-30) \mathrm{cm}$; en la plantación de Pinus radiata con un valor medio de $85,54 \mathrm{MgC} /$ ha con desviación estándar de $\pm 13,28$; mientras que el valor más bajo se observa en el estrato de páramo con un valor de $65,01 \mathrm{MgC} / \mathrm{ha}$ y una desviación estándar de 9,22; estudios similares (Ibrahim et al., 2007), reportan valores entre 52,3 $\pm 9,8$ y $81,3 \pm 3,6 \mathrm{MgC} / \mathrm{ha}$ en pasturas, lo que difiere de los ecosistemas de páramo por ser un sumidero de carbono.

Tabla 5. Contenido de carbono en el suelo. * Media \pm Desviación estándar

\begin{tabular}{ccccc}
\hline Conglomerado & Parcela & \multicolumn{3}{c}{ Profundidad de la toma de muestras de suelo } \\
& & $(0-10 \mathrm{~cm})(\mathrm{Mg} / \mathrm{ha})$ & $(10-20 \mathrm{~cm})(\mathrm{Mg} / \mathrm{ha})$ & $(20-30 \mathrm{~cm})(\mathrm{Mg} / \mathrm{ha})$ \\
\hline \multirow{3}{*}{001} & P1 & 73,15 & 73,76 & 71,70 \\
& P2 & 71,67 & 74,61 & 69,95 \\
& P3 & 71,41 & 73,76 & 65,01 \\
\hline \multirow{2}{*}{002} & P1 & 85,55 & $83,45^{\star} \pm 10,30$ & $85,54^{*} \pm 13,28$ \\
& P2 & 94,35 & 99,04 & 98,19 \\
& P3 & $80,25^{*} \pm 9,22$ & 88,90 & 91,22 \\
\hline
\end{tabular}

\section{Carbono orgánico total en parcelas permanentes de muestreo (PPM)}

El contenido promedio de carbono en la biomasa aérea en la plantación forestal fue de $(14,69 \mathrm{MgC} / \mathrm{ha})$, sus límites de confianza $+18,63 ;-10,7$. En la tabla 6 muestra la cantidad de carbono presente en los CUTs, evaluados. Como se esperaba en la plantación de pino fue donde se logró el mayor secuestro de carbono con, $(287,31 \mathrm{MgC} / \mathrm{ha})$ en comparación con el observado en el páramo $(277,82 \mathrm{MgC} / \mathrm{ha})$.

Tabla 6. Contenido de carbono orgánico total presente en los cinco depósitos. ${ }^{*}$ Media \pm Desviación estándar

\begin{tabular}{|c|c|c|c|c|c|c|c|c|}
\hline $\begin{array}{l}\text { Conglo } \\
\text { merado }\end{array}$ & CUTs & Parcela & $\begin{array}{c}\text { Biomasa } \\
\text { aérea } \\
\mathrm{MgC} / \mathrm{ha}\end{array}$ & $\begin{array}{l}\text { SOTB } \\
\mathrm{MgC} / \mathrm{ha}\end{array}$ & $\begin{array}{l}\text { DNV } \\
\mathrm{MgC/ha}\end{array}$ & $\begin{array}{l}\text { Raicillas } \\
\mathrm{MgC/ha}\end{array}$ & $\begin{array}{l}\text { Suelo } \\
\mathrm{MgC} / \mathrm{ha}\end{array}$ & $\begin{array}{c}\text { Carbono Total } \\
\mathrm{Mg} / \mathrm{ha}\end{array}$ \\
\hline \multirow{3}{*}{001} & \multirow{3}{*}{ Páramo } & P1 & & $6,48^{*} \pm 0,31$ & 50,46 & 0,15 & 218,61 & $275,70^{*} \pm 4,72$ \\
\hline & & P2 & & 6,29 & 51,84 & $0,17^{\star} \pm 0,02$ & $216,23^{*} \pm 4,35$ & 274,53 \\
\hline & & P3 & & 6,89 & $65,96^{\star} \pm 8,58$ & 0,19 & 210,17 & 283,22 \\
\hline \multirow{3}{*}{002} & \multirow{3}{*}{$\begin{array}{l}\text { Plantación } \\
\text { forestal }\end{array}$} & $\mathrm{P} 1$ & $16,33^{*} \pm 3,18$ & 0,39 & 3,52 & $0,16^{*} \pm 0,04$ & 254,54 & 274,93 \\
\hline & & P2 & 11,03 & 0,93 & 2,66 & 0,10 & 291,59 & 306,31 \\
\hline & & P3 & 16,72 & $0,71^{*} \pm 0,27$ & $2,72^{*} \pm 0,48$ & 0,17 & $260,37^{*} \pm 19,92$ & $280,69^{*} \pm 16,7$ \\
\hline
\end{tabular}

El contenido promedio de carbono en la biomasa aérea (Ruiz-Peinado et al., 2013), en la plantación forestal fue de 14,69 MgC/ha, sus límites de confianza +18,63 y -10,7; los mismos que están dentro del rango 20,3 y $79,7 \%$ respectivamente (Lazore y Gilabert, 2007), además se hace énfasis en el desarrollo de la plantación que varía con el tiempo pero a determinada edad el incremento se detiene. En potreros de Oaxaca se 
encontraron de 99 a $181 \mathrm{MgC} / \mathrm{ha}$ (Etchevers et al., 2002). Para el depósito de sotobosque se obtuvo el valor más alto en el estrato de páramo con una media de $6,55 \mathrm{Mg} \mathrm{C} /$ ha y una desviación estándar de 0,31; mientras que para la plantación forestal se obtuvieron los valores de $0,68 \mathrm{Mg} \mathrm{C} / \mathrm{ha}$ con una desviación estándar de 0,27; reportes similares (Ruiz, 2002), mencionan valores de (12,5 $\pm 3,6 \mathrm{MgC} / \mathrm{ha}$ ); la cantidad de carbono acumulado en la materia muerta o detritus no vivo obtuvo el valor más alto en el estrato de páramo con un valor medio de 56,09 $\mathrm{Mg} \mathrm{C} / \mathrm{ha}$, con una desviación estándar de 8,58, mientras que la plantación forestal tiene un valor medio de 2,97 Mg C/ha, con una desviación estándar de 0,48; (Gasparri y Manghi, 2004), reporta valores de $6,92 \mathrm{Mg} \mathrm{C} /$ ha para madera muerta y $2,80 \mathrm{Mg} \mathrm{C} / \mathrm{ha}$, para hojarasca.

Los resultados obtenidos en páramo y plantación forestal fueron similar es a los obtenidos por (Gayoso y Schlegel, 2001), quien registró estimaciones de 389 y $212 \mathrm{Mg} / \mathrm{ha}$, en bosques ubicados en la cordillera de los Andes y de la costa de Chile respectivamente.

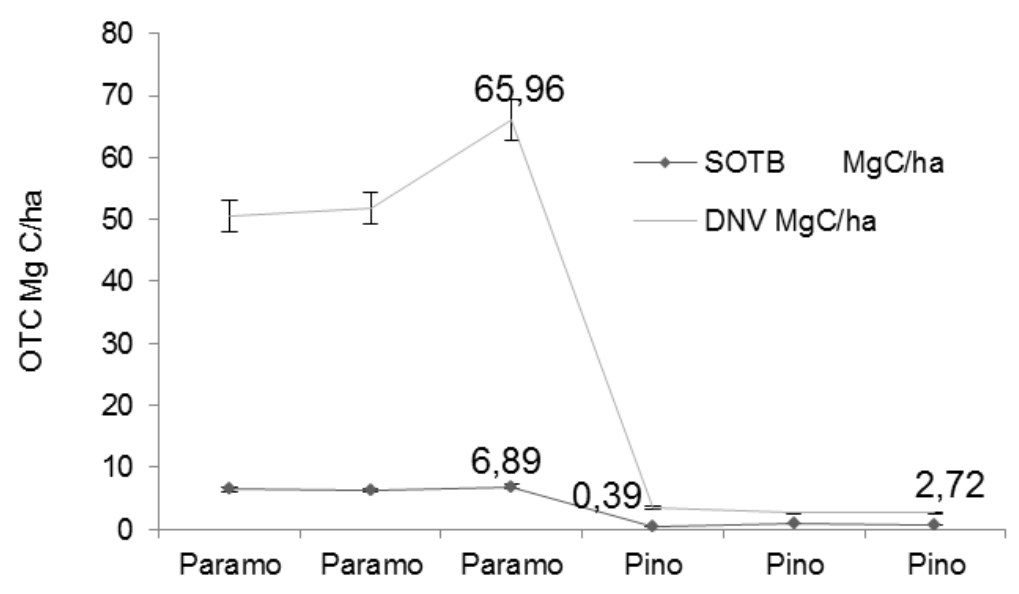

Fig. 5: Relación del carbono orgánico en SOTB/DNV

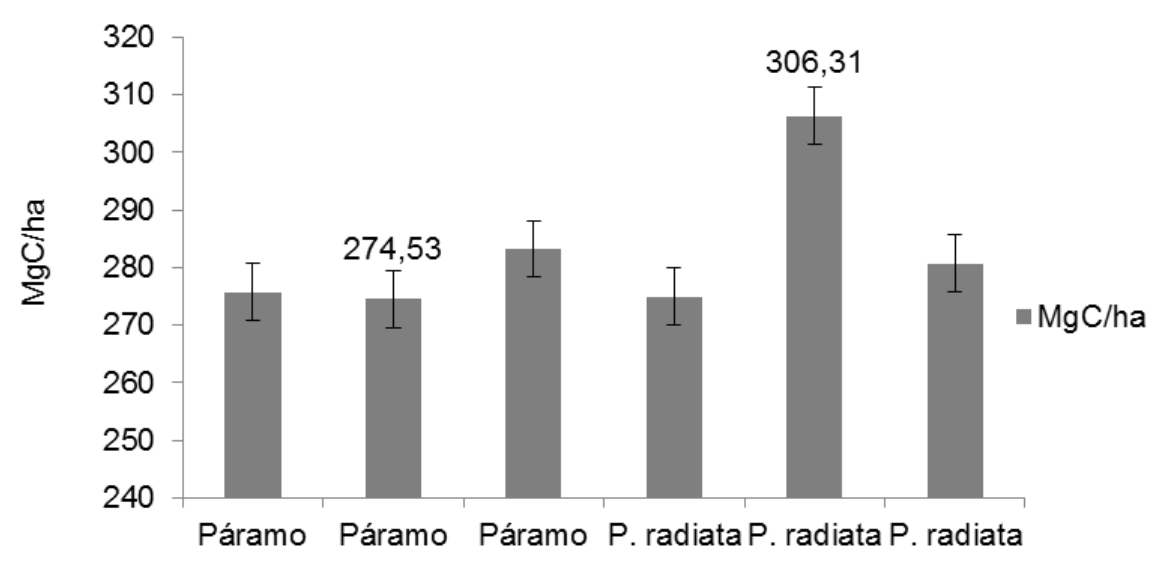

Fig. 6: Carbono orgánico total

Varios estudios han estimado la acumulación de biomasa total aérea y la consecuente fijación de carbono en bosques no intervenidos (Krankina et al., 1996), plantaciones forestales y bosques secundarios usando 0 desarrollando modelos estadísticos, (Brown y Lugo, 1982; Brown et al., 1989; Brown and Lugo, 1990; Brown, and Lugo, 1992; Brown, 1997; Nelson y Sommers, 1996; Dauber, 1995; Schlesinger, 2000). El carbono orgánico del suelo (Martínez et al., 2008), se encuentra en forma de residuos orgánicos poco alterados de vegetales, animales y microorganismos, en forma de humus y en formas muy condensadas de composición próxima al carbono elemental (Céspedes, 2012).

Como se puede observar, los cálculos de la captura de carbono en plantaciones forestales son muy sensibles a los cambios en la productividad. En el segundo grupo de variables en cuanto a su sensibilidad están la cantidad del carbono en el suelo, la densidad de la madera y el factor de expansión para calcular la biomasa total del árbol. 
Los suelos ricos en materia orgánica (Effron et al., 2006), tienen mayor porosidad y por lo tanto retienen un mayor volumen de agua; a medida que la densidad aparente aumenta, se reduce la porosidad del suelo y por lo tanto el contenido orgánico disminuye (Chen y Twilley, 2005; Cuevas et al., 2006). El presente estudio muestra una mayor concentración de carbono en el suelo, estos resultados concuerdan con lo reportado por (Swift, 2001), en la que menciona que en este tipo de depósito contienen más carbono que la suma existente en la vegetación y en la atmósfera.

\section{CONCLUSIONES}

Los factores de expansión es una herramienta útil, tomando en cuenta el impacto que genera tomar muestras destructivas al realizar el análisis del contenido de carbono que retienen las plantaciones forestales en proyectos de conservación y servicios ambientales.

La proporción entre la biomasa aérea con respecto a la biomasa subterránea muestra una tendencia proporcional del crecimiento de la (raíz/fuste) es del 21 y $79 \%$ respectivamente presente en todas las clases diamétricas el crecimiento dependerá de factores externos ya mencionados, y del manejo técnico que se implemente en la plantación. El contenido de carbono acumulado en suelo no es inversamente proporcional con respecto a la profundidad ya que en el páramo existen grandes depósitos de "turba" o materia orgánica en descomposición esto permite considerar que el ecosistema de paramo es un gran depósito de carbono.

El contenido de carbono en sotobosque y detritus no vivo es mayor en el ecosistema de páramo, debido a que en estos lugares existe regeneración natural, mientras que en una plantación forestal la pérdida de biodiversidad es alta por la perturbación del medio y degradación de servicios ecosistémicos.

En la plantación de pino insigne (Pinus radiata), fue donde se encontró el mayor contenido de carbono total con un valor medio de, $(287,31 \mathrm{MgC} / \mathrm{ha})$, un coeficiente de variación de 5,81 y un error relativo del $5 \%$, mientras que los datos obtenidos en el estrato de páramo fueron de $(277,82 \mathrm{MgC} / \mathrm{ha})$, un coeficiente de variación de 4,71 y un error relativo del 1,49\%.

\section{AGRADECIMIENTO}

Al SENESCYT por haber considerado al proyecto código 200, como uno de los ganadores en la convocatoria 2010 y al Vicerrectorado de Postgrado e Investigación de la Universidad Nacional de Chimborazo (UNACH), en la persona de la Dra. Ana Ríos, la cual hizo posible el desarrollo de esta investigación.

\section{REFERENCIAS}

Brown, S. y Lugo, A., The storage and production of organic matter in tropical forests and their role in the global carbon cycle. Biotropica. 14: 161-187 (1982).

Brown, S., Gillespie, A. y Lugo, A., Biomass estimation methods for tropical forests with applications to forest inventory data. Forest Science, 35:881-902 (1989).

Brown, S. y Lugo, A., Tropical secondary forest. Journal of Tropical Ecology 6: 1-32 (1990).

Brown, S. y Lugo, A., Above ground biomass estimates for tropical moist forests of the Brazilian Amazon. Interciencia 17:8-18 (1992).

Carvajal A., Feijoo, A., Quintero, H., y Rondón, M., Carbono orgánico del suelo en diferentes usos del terreno de paisajes Andinos Colombianos. Cienc. Suelo Nutr. / J. Soil. Sci. Plant. Nutr 9(3), 222-235 (2009).

Céspedes Flores, F. E., Fernández, J. A., Gobbi, J. A. y Bernardis, A. C. Reservorio de carbono en suelo y raíces de un pastizal y una pradera bajo pastoreo. Revista fitotecnia mexicana, 35(1), 79-86 (2012).

Chávez, Y., Rodríguez, G., Carrillo, C., Enríquez, R., Chávez, L. y Campos, V., Factores de expansión de biomasa aérea para Pinus chiapensis (Mart.) Andresen. Ciencias Agrícolas, 1273-1284 (2013).

Chen, R., y Twilley, R., A simulation model of organic matter and nutrient accumulation in mangrove wetland soils. Biogeochemistry. 44(1): 93-118 (2005). 
Concha, J., Alegre, J. y Pocomucha V., Determinación de las reservas de carbono en la biomasa aérea de sistemas agroforestales de teobroma cacao en el departamento de San Martìn, Perú. Ecología Aplicada, vol. 6, núm. 1-2, diciembre, 2007, pp. 75-82 (2007).

Coria, I., y De La Vega, M., Determinación de la Efectividad de la Remediación de Suelos Accidentalmente Contaminados con Ácido Sulfúrico mediante Métodos Geofísicos. Información Tecnológica. La Serena, v. 22, n. 1 (2011).

Cueva K. y otros 6 autores, Manual de Campo proyecto Evaluación Nacional Forestal y el programa nacional conjunto ONU - REDD+ del Ministerio del Ambiente del Ecuador. Bajo la cooperación del Programa "Manejo Forestal Sostenible ante el Cambio Climático" FAO Finlandia y el componente ONU REDD FAO (2012).

Cuevas, J., Seguel, O., Ellies A., Dorner J., Efecto de las enmiendas orgánicas sobre las propiedades físicas del suelo con especial referencia a la adición de lodos urbanos. Revista de la Ciencia del Suelo y Nutrición Vegetal. 6(2): 1-12 (2006).

Effron, D; Sarti, G., Quinteros, M. y Catan, S., Influencia de Especies Arbóreas Implantadas sobre Parámetros Biológicos y Bioquímicos en un Suelo Forestal de Chubut, Argentina. Información Tecnológica, vol.23, n.2 (2012).

Etchevers, J., Vargas, M., Acosta, y Velázquez, A., Estimación de la Biomasa Aérea Mediante el Uso de Relaciones Alométricas en Seis Especies Arbóreas en Oaxaca, México. Agrociencia Vol. 36 № 006. pp. 725 -736 (2002).

Fabre, V., Bizzotto, M., Tirner, J., Comportamiento Resistente de Suelos Orgánicos Estabilizados con Tanino. Información Tecnológica, La Serena, v. 21, n. 2 (2010).

Guerra, C., Gayoso, J., Schlatter, J. y Nespolo, R., Análisis de la biomasa de raíces en diferentes tipos de bosques: Avances en la evaluación de Pinus radiata en Chile. Bosque Valdivia. vol.26, n.1, pp. 5-21 (2005).

Hernández, G., Izquierdo, I., Alguacil, M., Caravaca F., y Roldán A. Captura de Carbono e indicadores de rehabilitación de suelos de áreas degradadas por la minería en MOA (Cuba). TOMO II, 505-514, Diputación de Badajoz, España (2006).

Hofstede, R. y Mena, P., Los beneficios escondidos del páramo: servicios ecológicos e impacto humano. Ecociencia Foundation; Mountain Institute; University of Amsterdam, Quito (2003).

Houghton, R., Converting terrestrial ecosystems from sources to sinks of carbon. Ambio Vol. 25, No. 4 (1996).

Ibrahim, M. y otros 7 autores, Almacenamiento de carbono en el suelo y la biomasa aérea en sistemas de uso de la tierra en paisajes ganaderos de Colombia, Costa Rica y Nicaragua. Agroforestería en las Américas, 45: 27-36 (2007).

Kyrklund, B., Cómo pueden contribuir los bosques y las industrias forestales a reducir el exceso de anhídrido carbónico en la atmósfera. Unasylva 163(41):12-14 (1990).

Krankina, O., Harmon, M., Winjum, K., Carbon storage and sequestration in Russian Forest Sector. Ambio 25: 284-288 (1996).

Lazore, A., Gilabert, P., Estimación del Carbono Capturado en las Plantaciones de Pino Radiata y Eucaliptos. Relacionadas con el DL-701 (2007).

Luteyn, J. L., y Churchill, S. P., Páramos: a checklist of plant diversity, geographical distribution, and botanical literature (p. 84). New York: New York Botanical Garden Press (1999).

Martínez E., Fuentes J., Acevedo E., Carbono Orgánico y Propiedades del suelo. Universidad de Chile. Facultad de Ciencias Agronomicas y Forestales. Plant Nutr. 8-1-68-96 (2008).

Mascha, J. y otros 5 autores, Significance of Over-Mature and Decaying Trees for Carbon Stocks in a Central European Natural Spruce Forest. Ecosystems 16: 336-346 (2013). 
Masera, O., Carbon mitigation scenarios for Mexican forests: methodological considerations and results. Interciencia 20(6): 388-395 (1995).

Návar, J., Allometric equations for tree species and carbon stocks for forests of northwestern Mexico. Forest Ecology and Management, 257(2), 427-434 (2009).

Nelson, D., Sommers LE. Total carbon, organic carbon, and organic matter. In: Sparks DL (ed) Methods of soil analysis. Part 3. Chemical methods; SSSA Book Series No. 5. Soil Science Society ofAmerica,Madison,Wisconsin, pp 961-1010 (1996).

Pauli, H., Gottfried, M., and Grabherr, G., Effects of climate change on the alpine and nival vegetation of the Alps. J. M. E. Journal of Mountain Ecology 7: 9-12 (2003).

Ravindranath, N., y Oswald, M., Carbon Inventory Methods: Handbook for Greenhouse Gas Inventory, Carbon Mitigation and Roudwood Production Projects. Springer (2008).

Ruiz, A., Fijación y almacenamiento de carbono en sistemas silvopastoriles y competividad económica en Matiguás, Nicaragua. Turrialba, CR, CATIE. 111 p (2002).

Ruiz, R., Bravo, A., Lopez, E., Montero G., Rio, M., Do thinnings influence biomass and soil carbon stocks in Mediterranean maritime pinewoods. Eur J Forest Res 132:253-262 (2013).

Sampaio, E., Estudio de las Prácticas Culturales en sus Relaciones con Agricultura, Suelo y Ambiente. Información Tecnológica, La Serena, v. 20, n. 3, (2009).

Sánchez, R., Ramos, R., Geissen, V., Mendoza, J., De la Cruz, E., Salcedo, E., Palma, D., Contenido de carbono en suelos con diferentes usos agropecuarios en el trópico Mexicano. Terra Latinoamericana, vol. 29, núm. 2, pp. 211-219 (2011).

Schlesinger, W., Carbon sequestration in soils: some cautions amidst optimism. Agriculture, Ecosystems and Environment, 82, 121-127 (2000).

Silva, M. y Navar, J., Estimación de factores de expansión de carbono en comunidades forestales templadas del norte de Durango, México. Rev. Chapingo, vol.15, n.2, pp. 155-160. ISSN 0186-3231 (2009).

Swift, R., Sequestration of carbon by soil. Soil Sci., 166, 858-871 (2001).

Torres, J., Espinoza, W., y Reddiar, L., y Vázquez, A., Secuestro de carbono en potreros arbolados, potreros sin árboles y bosque caducifolio de Huatusco, Veracruz. Tropical and Subtropical Agroecosystems, 13(3), 543-549 (2011).

Vesterdal, L. y otros 5 autores, Carbon sequestration in soil and biomass following afforestation: experiences from oak and Norway spruce chronosequences in Denmark, Sweden and the Netherlands. In Environmental Effects of Afforestation in North-Western Europe (pp. 19-51). Springer Netherlands. (2007). 